\title{
B-Lines on Chest Ultrasound Predicts Elevated Left Ventricular Diastolic Pressures
}

\author{
Maadarani $\mathrm{O}^{*}$, Bitar $\mathrm{Z}^{2}$, and Almeri $\mathrm{K}^{3}$ \\ ${ }^{1}$ Consultant Cardiologist, Internal medical department, Ahmadi hospital, Kuwait Oil Company, Kuwait \\ ${ }^{2}$ Consultant physician and intensivist, Internal medicine department, Ahmadi hospital, Kuwait Oil Company, Kuwait \\ ${ }^{3}$ Consultant interventional cardiologist, Chest disease hospital, Kuwait
}

${ }^{*}$ Corresponding author: Maadarani O, Consultant Cardiologist, Internal medical department, Ahmadi hospital, Kuwait Oil Company, Kuwait, Tel: 0096566986503, E-mail: ossamamaadarani@yahoo.com

Citation: Maadarani O, Bitar Z, Almeri K (2017) B-Lines on Chest Ultrasound Predicts Elevated Left Ventricular Diastolic Pressures. J Clin Exp Res Cardiol 3(2): 205

Received Date: September 18, 2017 Accepted Date: December 27, 2017 Published Date: December 29, 2017

\begin{abstract}
Background: Echocardiography and lung ultrasound are important tests for assessing left ventricular function in patients presented to the emergency department with acute pulmonary edema. Chest ultrasound is becoming an important tool in diagnosing acute pulmonary edema.

Aim: To investigate the relationship between the B profile on ultrasound chest and Spectral tissue Doppler echocardiography (E/e' ratio) in patients presented with the suspicion of acute pulmonary edema.

Methods: This paper reports a prospective observational study of 61 consecutive patients, which was presented with symptoms and signs of pulmonary edema and B - profile detected by echocardiography with a $5 \mathrm{MHz}$ curvilinear probe. Critical care physicians trained in ultrasound examination performed echocardiography and chest ultrasounds.

Results: Sixty-one participants were included in the study. Forty-seven of the 61 patients had a B-profile and 14 patients had an A profile. The mean E/e' level in the patients with B-profile was 20.8, compared with the mean level in the patients with an A-profile of $8.2(\mathrm{CI}=0.33-0.82)$. The distribution in the two groups differed significantly $(\mathrm{p}=0.003)$. Based on the value of $\mathrm{E} / \mathrm{e}$, the sensitivity and specificity were determined; the sensitivity of B profile on ultrasound was $92 \%$ ( $95 \%$ confidence interval $(\mathrm{CI})=0.812-0.968)$, and the specificity was $91 \%(\mathrm{CI}=0.623-0.98)$. The positive predictive value of the $\mathrm{B}$-profile was $97 \%(\mathrm{CI}=0.889-0.996)$, and the negative predictive value was $71 \%(\mathrm{CI}=0.454-0.883)$. The systolic function in the subjects with a B-profile was below $50 \%$ in $74.3 \%$ of the subjects and normal in $25.7 \%$ of the subjects. All the subjects with B profile and systolic function $>50 \%$ had elevated ProBNP and E/e' $>15$. An A-profile subjects had systolic function $>55 \%$.
\end{abstract}

Conclusions: Detecting the B-profile in lung ultrasound is highly sensitive and specific for elevated left ventricular diastolic pressures regardless of the systolic function of the left ventricle which may help in diagnosing pulmonary edema.

Keywords: B -lines; Ultrasound chest; Spectral tissue Doppler

\section{Background}

Acute pulmonary edema is a common problem facing emergency department (ED) physicians, and a percentage of these patients are admitted to the coronary care unit (CCU). The diagnosis of acute pulmonary edema remains a challenge for the following reasons: the presentation could be in combination with other diseases, such as chronic obstructive airway disease; and these diseases may have a presentation that is similar to that of acute pulmonary edema.

Cardiologists and intensivists commonly assess the heart using echocardiography. To save time, an extended evaluation could be performed using the same probe to complete the evaluation without changing the probe. Chest ultrasound is used to detect subpleural interstitial edema lines (B-lines) and pleural effusion.

The assessment of left ventricle diastolic function and filling pressures is of paramount clinical importance to distinguish heart failure (especially heart failure with preserved ejection fraction- (HFPEF)) from other diseases such as pulmonary disease resulting in acute dyspnea.

The ratio of E/e' is used to estimate left ventricle filling pressure (LVFP) and its use is recommended by the American Society of Echocardiography (ASE) and European Society of Cardiology (ESC) for evaluating diastolic dysfunction (DD) and HFpEF [4,5].

A B-line is a discrete, laser-like, vertical, hyperechoic image that arises from the pleural line. The B-lines are useful for the differential diagnosis of cardiogenic versus non-cardiogenic dyspnea [3]. 
The assay for plasma ProBNP is a useful test for the evaluation of patients with dyspnea, and it is particularly useful as a component of the evaluation of a suspected heart failure when the diagnosis is uncertain [1]. Hence, the study aim to investigate the relationship between the B profile on ultrasound chest and Spectral tissue Doppler echocardiography (E/e' ratio) in patients presented with the suspicion of acute pulmonary edema.

\section{Methods}

\section{Study design}

This work was a prospective, blinded observational study of an enrolled sample of emergency department (ED) patients in whom the ED physician requested N-terminal pro-B-type natriuretic peptide (NT-proBNP).

Population and setting: The study was performed in the Ahmadi hospital ED and CCU. The hospital is a subsidiary of Kuwait Oil Company (KOC). The hospital serves KOC members and their families, including their parents, with annual ED census of 60,000 visits. US examinations are currently used in daily practice in the ED, CCU and Intensive care unit (ICU) of this hospital.

Patients were included if they were $>18$ years old and had acute dyspnea, and if the treating ED physician's clinical suspects that acute pulmonary edema and left ventricular failure was part of the differential diagnosis after the history and physical examination and before any testing was completed or a serum NT-ProBNP was ordered in the ED. We excluded patients if they were non-KOC members, because the follow up would be in other hospitals in Kuwait. Additionally, we excluded patients who ultrasound profile showed another pattern other than A \& B and who were incapable of providing informed consent.

Protocol: Each patient underwent an 8-zone thoracic ultrasound. The treating physician in the ED obtained informed written consent as well as baseline demographic and clinical data. Ultrasound scans were performed by a physician trained in the techniques of chest ultrasound and echocardiography. The ultrasound images were saved to a hard drive and reviewed in the system by a senior intensives trained in critical care medicine. The physicians were blinded to the E/e' and NT-ProBNP results.

Ultrasound was performed using portable echocardiography (GE Vivid S6N, N-3191Horten, and Norway) equipment with a $5-\mathrm{MHz}$ broadband curvilinear transducer in the echocardiography preset.

The exams consisted of bilateral scanning of the anterior and lateral chest wall and were performed with the patients in the supine or near-to-supine position. The correct scan was intercostal with the maximum extension of the visible pleural line. The chest wall was divided into 8 areas, and scans for each area were obtained. The areas included two anterior and two lateral regions per side. The anterior chest wall was delineated from the sternum to the anterior axillary line and was subdivided into upper and lower halves (approximately from the clavicle to the second-third intercostal spaces and from the third intercostal space to the diaphragm). The lateral zone was delineated from the anterior to the posterior axillary line and was subdivided into upper and basal halves. The probe was placed in a cephalic orientation, and the pleural line was placed in the middle of the image by adjusting the depth settings [2].

Measurements: The two primary findings on thoracic ultrasound are A-lines and B-lines. A B-line is a comet-tail artifact that arises from the pleural line and moves in concert with lung-sliding. It is long, well-defined, laser-like, and hyperechoic and it erases A-lines (2). The updated definition of B-line requires three constant criteria (comet tail arising from pleural line and moving with lung sliding) and four quite constant criteria (long, well-defined, hyperechoic and erasing A-line) [3]. An A-line is the repetition of the pleural line, and it is an approximately horizontal hyperechoic line parallel to the pleural line [2]. Two important profiles are detected, as follows: The A-profile (Figure 2) associates anterior lung sliding with A-lines, and the B-profile (Figure 1) is defined by the presence of three or more B-lines in a longitudinal plane between two ribs per scan area, diffuse B lines in more than one scan per side, and the presence of B lines in both sides associates with anterior lung sliding [4].

Echocardiography method: Echocardiography is the mainstay of the non-invasive evaluation and quantitation of diastolic function [4,5]. Myocardial stiffness and relaxation abnormalities in diastolic dysfunction (DD) result in elevated left ventricular filling pressure(LVFP) that is indirectly evaluated with echocardiography [4,5]. Early mitral annular velocity (e') obtained with the use of tissue Doppler imaging provides an assessment of LV myocardial relaxation. In conjunction with mitral peak early filling velocity E, the ratio of E/e' is used to estimate LVFP and its use is recommended by the American Society of Echocardiography (ASE) and European Society of Cardiology (ESC) for evaluating diastolic dysfunction (DD)/ heart failure with preserved ejection fraction (HFpEF) [4,5] (Figure 3). E/e' lateral $>12$, E/e' mean $>13$, or E/e' septal $>15$ indicates elevated LVFP, whereas E/e' $<8$ (any location) indicated normal LVFP [4]. When E/e' falls into the intermediate zone $\left(8>\mathrm{E} / \mathrm{e}^{\prime}<12-15\right)$, lung ultrasonography was incorporated into the study to estimate left atrial pressure (LAP), which is considered positive when US chest shows B profile and elevated Pro BNP [5-7].

These velocities are recorded from the apical four-chamber view by placing a 5-mm to 6-mm sample volume over the lateral or medial portion of the mitral annulus to cover the longitudinal excursion of the mitral annulus in both systole and diastole. The velocity scale is set at about $20 \mathrm{~cm} / \mathrm{sec}$ above and below the zero-velocity baseline; the angulation between the ultrasound beam and the plane of cardiac motion was placed to be minimal. The recommendation for spectral recordings is a sweep speed of 50 to $100 \mathrm{~mm} / \mathrm{sec}$ at end expiration, and measurements are averaged for at least three consecutive cardiac cycles [1]. 


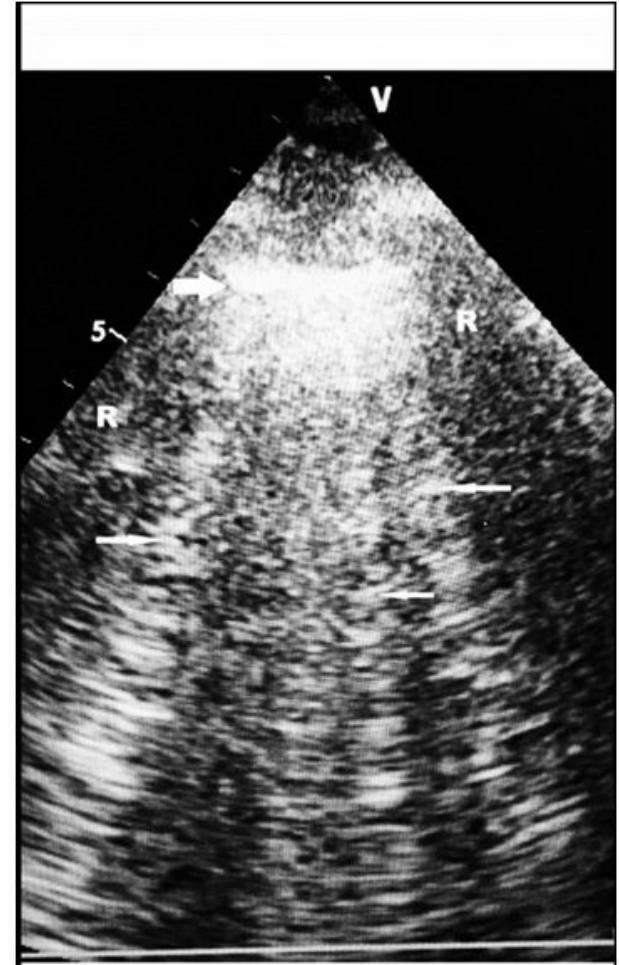

Figure 1: B lines Thick arrow-Pleural line Thin arrow-B lines R-Rib

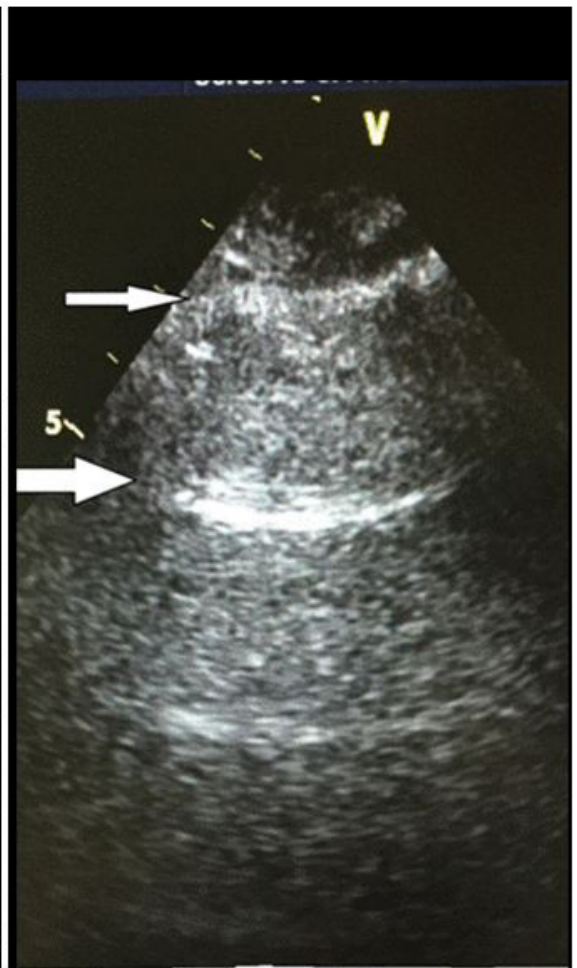

Figure 2: A lines Thin arrow-Pleural line Thick arrow-A line: a horizontal artifact

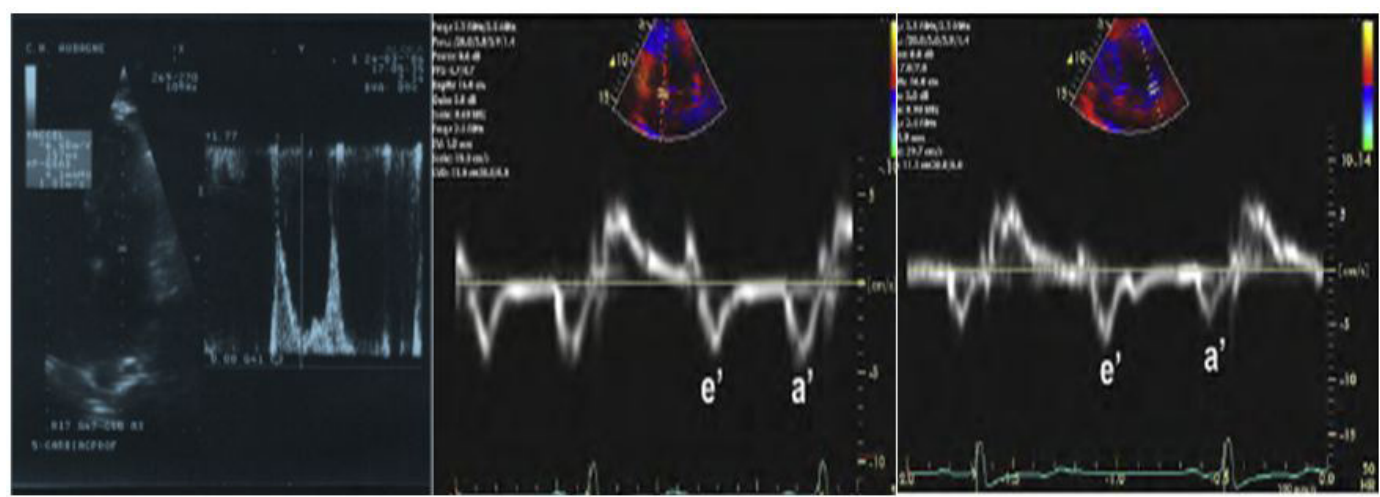

Figure 3: Septal (middle) and lateral (right) tissue Doppler early (e') and late (a') diastolic velocities are markedly reduced with Average E/e' ratio is $>14$, consistent with elevated left end diastolic pressure

The analysis was performed offline by two independent investigators experienced in echocardiographic measurements that were blinded to hemodynamic data at the time of analysis. All reported echocardiographic measurements were averaged from 3 consecutive cycles. LV volumes, LV ejection fraction, mitral regurgitation, and left atrial maximum volume were assessed as recommended by the ASE (1). Mitral inflow was analysed for peak E (early diastolic) and peak A (late diastolic) velocities, E/A ratio, and deceleration time of E velocity.

Data analysis: The statistical analyses were performed using SPSS 19. We determined the median E/e' value for patients with A-profile and B- profile, and calculated 95\% confidence intervals (CI) of the differences. The evaluation of the data was performed with the Mann-Whitney $U$ test to delineate the significance of the difference in the rank order of E/e' in patients with A-profile and B-profile.

The NT ProBNP level was considered positive at the threshold limit in relation to age. For age $<50$, the threshold limit for being positive was $450 \mathrm{pg} / \mathrm{mL}$, for age 50 to 75 , the level was $900 \mathrm{pg} / \mathrm{mL}$, and for age $>75$, the level was $1800 \mathrm{pg} / \mathrm{mL}$. A level below 300pg/ $\mathrm{mL}$ excludes a diagnosis of heart failure with a negative predictive value of $80 \%$ [8].

\section{Results}

Sixty-one participants were included in the study. The mean age was 66.8 years, with a range of 44 to 93 years; $49.2 \%$ of the subjects were male. A total of forty-seven of the 61 patients had B- profile and hemodynamic pulmonary edema. The remaining 14 patients of had A- profile. The clinical characteristics of the patients in relation to ultrasound chest profiles are shown in Table 1. 


\begin{tabular}{|l|c|c|c|c|}
\hline & A Profile & B Profile & Total & P \\
\hline \multicolumn{1}{|c|}{ Medical history } & No (\%) & No(\%) & No(\%) & \\
\hline Myocardial infarction & & & & \\
\hline Angina & $0(0.0)$ & $37(72.5)$ & $37(60.7)$ & $<0.0001$ \\
\hline Coronary artery bypass Graft & $3(30.0)$ & $27(52.9)$ & $30(49.2)$ & 0.164 \\
\hline Atrial fibrillation & $0(0.0)$ & $12(23.5)$ & $12(19.7)$ & 0.091 \\
\hline Hypertension & $0(0.0)$ & $10(19.6)$ & $10(16.4)$ & 0.142 \\
\hline NIDDM & $6(60.0)$ & $45(88.2)$ & $51(83.6)$ & 0.049 \\
\hline IDDM & $7(70.0)$ & $45(88.2)$ & $52(85.2)$ & 0.99 \\
\hline Asthma & $3(30.0)$ & $6(11.8)$ & $9(14.8)$ & 0.157 \\
\hline Obstructive airway disease & $2(20.0)$ & $3(5.9)$ & $5(8.2)$ & 0.185 \\
\hline Chronic renal impairment & $4(40.0)$ & $6(11.8)$ & $10(16.4)$ & 0.49 \\
\hline \multicolumn{1}{|c|}{ Clinical examination } & $1(10.0)$ & $23(45.1)$ & $24(39.3)$ & 0.037 \\
\hline Shortness of breath & & & & \\
\hline Elevated jugular venous pressure & $2(14)$ & $33(70)$ & $35(57)$ & $<0.0001$ \\
\hline Pulmonary rales & $4(28)$ & $29(61.7)$ & $33(54)$ & $<0.0001$ \\
\hline Wheezing & $5(30)$ & $13(28)$ & $18(29.5)$ & 0.85 \\
\hline S3 gallop & $2(14)$ & $35(74.4)$ & $37(60.6)$ & $<0.0001$ \\
\hline Hypoxemia & $12(85.7)$ & $43(89)$ & $55(90)$ & 0.02 \\
\hline \multicolumn{1}{|c|}{ Chest radiograph findings } & & & & \\
\hline Normal heart size & $11(78.5)$ & $11(23.4)$ & $22(36)$ & $<0.0001$ \\
\hline Pulmonary venous congestion & $2(14)$ & $43(89)$ & $45(73.7)$ & $<0.0001$ \\
\hline Interstitial edema & $1(7)$ & $40(85)$ & $41(67)$ & $<0.0001$ \\
\hline Alveolar edema & $1(7)$ & $40(85)$ & $41(67)$ & $<0.0001$ \\
\hline Tale 1 Clican & $45(95.7)$ & $57(93.4)$ & 0.02 \\
\hline
\end{tabular}

Table 1: Clinical characteristics of the patients in relation to ultrasound chest profile

The analysis revealed that A -profile was present in all patients with normal E/e'ratio (E/e' of $<8$ ) and NT-ProBNP $<400$ or 300 pg/ $\mathrm{mL}$. Fifty of the 61 patients had elevated E/e' and ProBNP levels in relation to age, 46 patients had E/e' level > 15 (Table 2).

\begin{tabular}{|c|c|c|c|}
\hline Thoracic ultrasound profile & High E/e' & Normal E/e' & Total \\
\hline B- Profile & 46 & 1 & $\mathbf{4 7}$ \\
\hline A - profile & 4 & 10 & 14 \\
\hline Total & 50 & 11 & 61 \\
\hline Variable & Value & $\mathbf{9 5 \%}$ confidence interval \\
\hline Sensitivity & 0.92 & 0.812 to 0.968 \\
\hline Specificity & 0.91 & 0.623 to 0.98 \\
\hline Positive predictive value & 0.97 & 0.889 to 0.996 \\
\hline Negative predictive value & 0.714 & 0.454 to 0.883 \\
\hline LR+ & 10.12 & 1.559 to 65.697 \\
\hline LR- & 0.088 & 0.034 to 0.229 \\
\hline
\end{tabular}

(LR+: positive likelihood ratio, LR-: negative likelihood ratio)

E: peak E mitral velocity; e’: peak early diastolic velocity by spectral tissue Doppler at the septal or lateral side of mitral annulus

Table 2: Chest ultrasound profiles based on Spectral tissue Doppler echocardiography (E/e' ratio)

The median of E/e' levels in patients with B-profile was 20.8, compared with a median of 8.2 in the subjects with A-profile (P $=0.003 \mathrm{CI}=0.33-0.82$ ).

The distributions in the 2 groups differed significantly (Mann-Whitney U-static $=3.98$ ). Based on the threshold level of NTProBNP of 250 and significant elevated E/e', the sensitivity of detecting B-profile on ultrasound was $95.0 \%$, and the specificity was $92.0 \%$. The positive predictive value of the B-profile was $90.0 \%$, and the negative predictive value was $88.0 \%$ (Table 2 ).

The systolic function in the subjects with a B-profile was below $50 \%$ in $74.3 \%$ of the subjects and normal in $25.7 \%$ of the subjects. The subjects with an A-profile had a systolic function > 55\%. 
To increase the sensitivity of B-lines and chest ultrasound in cases of elevated E/e' to reach a diagnosis in patients with dyspnea, we included Framingham score for the diagnosis of heart failure and the ejection systolic function (EF) when performing the echocardiography.

Framingham criteria of heart failure requires the simultaneous presence of at least 2 major criteria or 1 major in conjunction with 2 minor criteria (major criteria: acute pulmonary edema, cardiomegaly on CXR, hepatojugular reflux, neck vein distension, paroxysmal nocturnal dyspnea, pulmonary rales, S3gallop, weight loss $>4.5 \mathrm{Kg}$ in 5 days in response to treatment; minor criteria: ankle edema, dyspnea on exertion, hepatomegaly, nocturnal cough, pleural effusion, tachycardia). The Framingham heart study criteria are $97 \%$ sensitive and $78 \%$ specific for identifying persons with congestive heart failure.

Of those subjects with a B-profile, $94 \%$ had a Framingham score of CHF $>4$; all the subjects with an A-profile had a score of $<4$, $\mathrm{p}<0.0001$.

\section{Discussion}

The clinical diagnosis of acute heart failure (HF) syndromes is challenging in the emergency care setting [9]. Steg PG et al, in the landmark "Breathing not Properly Multinational" study, the Framingham score was reported to be $85 \%$ sensitive and 58\% specific for the clinical diagnosis of congestive HF in a large, unselected patient population presenting with acute dyspnea [10]. Therefore, additional diagnostic methods are required in this clinical setting to accurately establish the diagnosis of acute congestive HF.

The brain natriuretic peptide (BNP), a cardiac neurohormone that is secreted in response to myocardial stretch and volume overload, has been validated as a powerful and cost-effective diagnostic marker of congestive HF [11], and is extensively utilized as the first-line diagnostic complement to clinical and radiographic data in the acute care setting. Detecting the B-profile is highly sensitive and specific for elevated NT-proBNP, which helps in diagnosing pulmonary edema. Performing chest ultrasound could be part of the echocardiography evaluation in patients with acute dyspnea [12].

The ratio of flow Doppler mitral E wave peak velocity and early diastolic velocity of the mitral annulus obtained by tissue Doppler (E/e') (a mean of the values obtained at the septum and the lateral wall) is a valuable tool for non-invasively determining LV diastolic pressures [13]. This ratio is related to pulmonary capillary wedge pressure and its variation is also related to the variation in capillary wedge pressure, although the correlation is not strong enough to allow the use of one variable instead of the other; nevertheless, it enables patients with elevated pulmonary capillary wedge pressure (defined as $>15 \mathrm{mmHg}$ ) to be identified accurately. Noninvasive assessment of LV diastolic pressures at presentation, before tailored therapy, is clinically relevant particularly for patients with severe symptoms, inconclusive clinical, radiographic, biochemical data and preserved LV ejection fraction at echocardiography [14]. Currently, the ASC recommends the use of color M-mode and spectral tissue Doppler-derived indexes, E/Vp and E/e' respectively, for assessing left atrial pressure in clinical trials [15]. Dokainish et al have first reported the diagnostic accuracy of tissue Doppler echocardiography in a large patient population referred for suspected congestive HF with a wide range of symptoms (NYHA class II to IV) and LV ejection fractions [16]. The diagnostic accuracy of E/e' was similar to BNP regardless of LV ejection fraction; furthermore, these 2 methods were able to provide independent diagnostic information, supporting their complementary role in this setting.

The usefulness of bedside tissue Doppler echocardiography as well as its incremental role over the clinical judgment and BNP testing in the emergency diagnosis of acute HFPEF in patients hospitalized for acute severe dyspnea is well documented and confirmed; this noninvasive method was found to be accurate, even among patients with inconclusive BNP levels (100-400 pg/ $\mathrm{ml}$ ) or arrhythmia $[17,18]$.

Measurement of mitral valve inflow and mitral annular velocity allows the intensivist to identify an elevated or normal left atrial pressure (LAP) in some cases, but may yield an indeterminate result. The ultrasonographer is then required to make a series of echocardiography measurements. Given the time constraints and difficult imaging conditions in the ICU, these are not practical for the frontline intensivist to perform. Instead, lung ultrasonography may be incorporated into the study to estimate LAP. While some data suggest an association between B-line number and right-sided pressures, other did not find a relationship with leftsided pressures as estimated by the PCWP [19]. Agricola et al. studied 20 patients (mean LVEF 64\%) before and after cardiac surgery and did see positive correlations between B-lines on lung ultrasound in 28 chest zones and PCWP $(r=0.48)$ [20]. A study of 72 patients (mean LVEF 41\%) undergoing stress echocardiography in which PCWP was estimated echocardiographically by tissue Doppler also found positive correlations between estimated PCWP and B-lines $(r=0.69)$ [21]. The discrepancy in results between these studies could be due to different size and type of study populations, the fact that there might be an association between B-lines and PCWP, but only in patients with acute decompensated heart failure [19].

In our study the median E/e' levels in patients with B-profile were 20.8 , compared with a median of 8.2 in the subjects with A-profile.

It was found that the sensitivity and the specificity of detecting B-profile on ultrasound is high when E/e'> 15 (95.0\% and $92.0 \%$ consecutively), which concluded the high correlation between finding B profile on U/S chest and elevated left ventricle filling pressure in a patient presenting with picture of suggestive of heart failure . 
Also it was noticed that most patients with A profile had normal E/e' ratio.

Our study was done only in a population with a suspicion of heart failure.

The limitation of our study was a single centre study with a relatively small sample size. As an ultrasound evaluation, lung ultrasound (LUS) shares all limitations related to an operator-dependent technique. It is also true that since the examination is much simpler than other ultrasound applications (i.e., echocardiography), the inter-operator variability is low.

\section{Conclusion}

The B-line assessment on chest ultrasound may be useful in assessing left-sided filling pressures in patients with suspicion of heart failure.

\section{References}

1. Raymond I, Groenning BA, Hildebrandt PR, Nilsson JC, Baumann M, et al. (2003) The influence of age, sex and other variables on the plasma level of N-terminal pro brain natriuretic peptide in a large sample of the general population. Heart 89: 745-51.

2. Volpicelli G, Elbarbary M, Blaivas M, Lichtenstein DA, Mathis G, et al. (2012) International liaison committee on lung ultrasound (ILC-LUS) for international consensus conference on lung ultrasound (ICC-LUS). Intensive Care Med 38: 577-91.

3. Lichtenstein DA (2014) Lung ultrasound in the criticallyill. Ann Intensive Care 4: 1-12.

4. Lichtenstein D, Mezière G (2008) Relevance of lung ultrasound in the diagnosis of acute respiratory failure: the BLUE protocol. Chest 134: 117-25.

5. Copetti R, Soldati G, Copetti P (2008) Chest sonography: a useful tool to differentiate acute cardiogenic pulmonary edema from acute respiratory distress syndrome. Cardiovasc Ultrasound 6: 10.1186/1476-7120-6-16.

6. Nagueh SF, Appleton CP, Gillebert TC, Marino PN, Oh JK, et al. (2009) Recommendations for the evaluation of left ventricular diastolic function by echocardiography. J Am Soc Echocardiogr 22: 107-33.

7. Levitov AB, Mayo PH, Vastardis L (2015) Echocardiographic Assessment of Left Ventricular Systolic And Diastolic Function, Critical Care Ultrasonography (2nd edn), McGrawHillGlobal Education Holdings, LLC.

8. Januzzi JL, van Kimmenade R, Lainchbury J, Bayes-Genis A, Ordonez-Llanos J, et al. (2006) NT-proBNP testing fordiagnosis and short-term prognosis in acute destabilized heart failure:an international pooled analysis of 1256 patients: the International Collaborative of NT-proBNP Study. Eur Heart J 27: 330-7.

9. Wang CS, Fitz Gerald JM, Schulzer M, Mak E, Ayas NT (2005)Does this dyspneic patient in the emergency department have congestive heart failure? JAMA 294: 1944-56.

10. Steg PG, Joubin L, McCord J, Abraham WT, Hollander JE, et al. (2005) B-type natriuretic peptide and echocardiographic determination of ejection fraction in the diagnosis of congestive heart failure in patients with acute dyspnea. Chest 128: 21-9.

11. Silver MA, Maisel A, Yancy CW, McCullough PA, Burnett JC, et al. (2004) BNP Consensus Panel: A clinical approach for the diagnostic, prognostic,screening, treatment monitoring, and therapeutic roles of natriuretic peptides in cardiovascular diseases. Congest Heart Fail 10: 1-30.

12. Bitar Z, Maadarani O, Almerri K (2015) Sonographic chest B-lines anticipate elevated B-type natriuretic peptide level, irrespective of ejection fraction. Ann Intensive Care 5: 10.1186/s13613-015-0100-x.

13. Chirillo F, Brunazzi MC, Barbiero M, Giavarina D, Pasqualini M, et al. (1997) Estimating mean pulmonary wedge pressure in patients with chronic atrial fibrillation from transthoracic Doppler indexes of mitral and pulmonary venous flow velocity. J Am Coll Cardio 130: 19-26.

14. Dabbah S, Reisner SA, Aronson D, Agmon Y (2006) Left ventricular filling hemodynamics in patients with pulmonary edema and preserved versus reduced left ventricular ejection fraction: a prospective Doppler echocardiographic study. J Am Soc Echocardiogr 19: 733-43.

15. Gottdiener JS, Bednarz J, Devereux R, Gardin J, Klein A, et al. (2004) Echocardiography American Society of Echocardiography recommendations for use of echocardiography in clinical trials. J Am Soc Echocardiogr 17: 1086-119.

16. Dokainish H, Zoghbi WA, Lakkis NM, Quinones MA, Nagueh SF (2004) Comparative accuracy of B-type natriuretic peptide and tissue Doppler echocardiography in the diagnosis of congestive heart failure. Am J Cardiol 93: 1130-5.

17. Arques S, Roux E, Sbragia P, Ambrosi P, Taieb L, et al. (2005)Accuracy of tissue Doppler echocardiography in the emergency diagnosis of decompensated heart failure with preserved left ventricular systolic function. Comparison with B-type natriuretic peptide measurement. Echocardiography 22: 657-64.

18. Arques S, Roux E, Sbragia P, Pieri B, Gelisse R, et al. (2006) Accuracy of tissue Doppler echocardiography in the diagnosis of new-onset congestive heart failure in patients with levels of B-type natriuretic peptide in the mid-range andnormal left ventricular ejection fraction. Echocardiography 23: 627-34.

19. Platz E, Lattanzi A, Agbo C, Takeuchi M, Resnic FS, et al. (2012) Utility of Lung Ultrasound in Predicting Pulmonary and Cardiac Pressures. Eur J Heart Fail 14: 1276-84.

20. Agricola E, Bove T, Oppizzi M, Marino G, Zangrillo A, et al. (2005) 'Ultrasound comettail images': a marker of pulmonary edema: a comparative study with wedge pressure and extravascular lung water. Chest 127: 1690-5.

21. Agricola E, Picano E, Oppizzi M, Pisani M, Meris A, et al. (2006) Assessment of stressinduced pulmonary interstitial edema by chest ultrasound during exercise echocardiography and its correlation with left ventricular function. J Am Soc Echocardiogr 19: 457-63. 


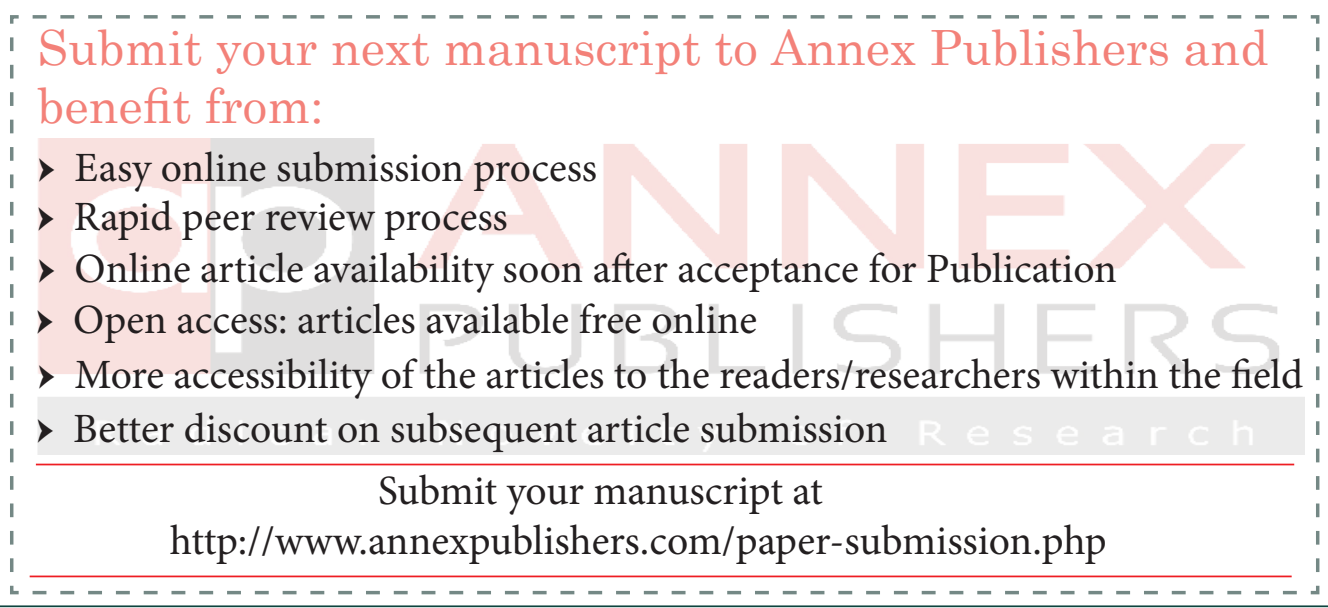

